\title{
A Biological WOCE
}

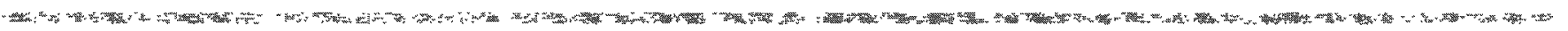

John A. McGowan

Scripps Institution of Oceanography $\bullet$ La Jolla, California USA

$\mathbf{P}$

attern recognition is the very basis on which we attempt to understand the world. This has two elements; patterns in space and patterns in time. In many ways these cannot be separated observationally, but as long as we are aware of the possible aliasing of our results we can use the appropriate observations to help us towards enlightenment. Describing spatial pattern in the ocean, especially the open ocean, is difficult to accomplish because of the vast space involved (read expensive ship-time here) but some preliminary such work has been done. The main confusion is that there are many scales of variability or what used to be called patchyness. Patchiness has not really received very much study, but never-the-less has served to obscure our understanding of which are the important scales of variability, diversity gradients and ecological boundaries, i.e. pattern recognition. A very similar, if not the same issue, was behind the World Ocean Circulation Experiment (WOCE) program in physical oceanography where it was called the signal to noise ratio problem. Eddies, fronts, and meanders served to alias our ability to identify major circulation systems whose dynamics were basically different and therefore our "understanding of the general circulation of the global ocean" was not good enough "to be able to model its present state and predict its evolution in relation to long term changes in the atmosphere." Resolution of these problems was accomplished by having very closely spaced stations in order to detect small scale variability but ships traveled long distances to enable detection of large spatial scale changes as well. That is, they studied many scales of spatial variability to allow spectral analysis to detect the "important" patterns. Because of the close station spacing and their desire to look at the entire water column, the number of properties routinely measured had to be rather few so that entire cross ocean transects could be accomplished in a short enough time that major temporal changes did not take place. I think we biologists should follow this lead.

But the design of the study very much depends on what the objectives are. I believe that the study of largescale species patterns are at the heart and soul of a new biological oceanography and modern ecology. Firstly, there is evidence that the number of species of sexually reproducing animals is far less than on land and the number of species of marine plants is a small fraction of the number of land plants. If so, why? We need better documentation of this from large areas of the ocean using modern taxonomy and molecular techniques. Are there many more species of which we are unaware? We have some knowledge of the large-scale patterns of diversity for some groups and it appears that the famous latitudinal gradient of diversity as shown on land is not so in the ocean. This, then, tests our "accepted" theories of the origins and maintenance of diversity. Why, if true, is the open ocean so different? There have been several studies of diversity maintenance in the ocean but these too challenge the theory, based on terrestrial studies. All of this needs much better documentation but is definitely worth doing. "The causes and conservation of Earth's diversity remain one of the greatest challenges facing ecology and society." (Tilman, 1999).

What and where are the major ecosystems of the open-ocean? There have been several approaches to this. Longhurst and Sherman (with his Large Marine Ecosystems, LMEs) have both written about divisions in the ocean but these are based on arbitrary criteria, not ecology or evolution but rather convenience. They are anything but objective. There are also more ecologically oriented studies based on species assemblages or community ecosystems. Here the composite spatial patterns of species of zooplankton and phytoplankton were used to detect where the organisms themselves reached a consensus on where important boundaries exist. The assumption is that the pattern of recurrent species groups represent coevolutionary adaptation to a suite of environmental variables, i.e. evolutionary development of organized community ecosystems, each with different dynamics and different ways of processing carbon. Preliminary but provocative studies show that such systems exist, can be detected with the proper design of sampling and tend to resemble in shape and number the major circulation regimes with their different physical dynamics. These different physical systems have apparently led to the evolution of different ecosystems.

All of this should be pursued if we are to make real progress and to make the most of this unique opportunity. But everything depends on sampling design and execution. The objective is to end up with quantitative, detailed maps of diversity and an outline of ecosystem species structure for the world's oceans. Maps must be good enough to allow one-for-one comparisons with 
the environmental maps produced by WOCE. If they "match" we've learned a great deal about how to proceed. That is, because of our new, very trustworthy, observations, we will be in a position to produce potentially disproveable null hypotheses for testing. In other words, we can now ask the question, "Why are things the way they are rather than some other way?" We cannot do this kind of science if our original observations on "the way things are," are non-existent or extremely dubious.

But more needs to be done than just catching fish. If large-scale surveys are done, it would be extremely wasteful to concentrate on one highly mobile group of organisms. A much more complete spectrum of the ecosystems should be quantitatively measured. This includes micro organisms, phytoplankton and zooplankton, as well as temperature, salinity (for comparison to WOCE), nutrients, particulate organic carbon (for fine scale biomass), acoustics (for coarse grain biomass), and optics. Many of these can be measured quite quickly and simply and most are done now as a matter of routine on California Cooperative Oceanic Fisheries Investigations (CalCOFI) cruises.

While the design and execution of measurement at sea is of great importance, a plan for the processing and analysis of the data is even more important. While many of the physical-chemical and gross biological measures (biomass for example) can be easily done, the determination of species diversity cannot.

There will be many hundreds of species, over a great size spectrum per station. These cannot be identified and enumerated with anything short of NASA's budget (not a bad idea at that) but a selected few categories can. These categories, if wisely chosen, can be used to describe the basic species and trophic patterns and serve as a baseline against which we can test modern ecological diversity origin and diversity maintenance theory, and to identify ecosystems and describe their structure.

But even this will be a major task. There have been long and frequent complaints in the past five or six years about the lack of experienced taxonomists and more explicitly the lack of decent jobs for young people who wish to do this kind of work. Universities and research institutes, NSF and NOAA are all at fault here; they will not support this work and as a result, now that diversity studies have been recognized to be so important, we have few authoritative experts. The Census of
Marine Life project can correct this glaring gap.

I suggest, as a part of this program, the endowment of five or six chairs of oceanic ecosystem taxonomy at several different institutions. These are for young persons trained in modern biology, ecology, and systematics and specifically linked to the sampling and measurements at sea. Their specialties must include more than just the highly mobile fish which are difficult to sample quantitatively, are not the best ecosystem indicators and where there is no shortage of taxonomists.

All of the above is only a brief rationale and outline of a plan but it is about some of the most pressing scientific problems facing society and, if carried out, can strongly influence or even change the direction of marine science for the next century.

\section{Suggested Readings}

McGowan, John A., 1974: The nature of oceanic ecosystems. In: The Biology of the Oceanic Pacific. Proceedings of the 33rd Annual Biology Colloquium. C.B. Miller, ed., Oregon State University Press, Corvallis, 9-28

McGowan, J.A. and P. Walker, 1993: Pelagic diversity patterns. In: Species Diversity in Ecological Communities, Historical and Geographical Perspectives. R.E. Ricklefs and D. Schluter, eds., University of Chicago Press, Chicago.

Reid, J.L., E. Brinton, A. Fleminger, E.L. Venrick, and J.A. McGowan, 1978: Ocean circulation and marine life. In: Advances in Oceanography. Proceedings of the General Symposium of the Joint Oceanographic Assembly, 13-24 September 1976, Edinburg Scotland, New York Plenum Press, 66-130.

Tilman, D., 1999: Diversity by default. Science, 283, 495496.

Venrick, E.L., 1990: Phytoplankton in our oligotrophic ocean: Species structure and interannual variability. Ecology, 7l, 1547-63. 